\title{
Reliability of Wrist Oscillometry Meter in Blood Pressure Measurements
}

\author{
Dr. Arturo L. Tapas Jr \\ Faculty, College of Engineering, AMA International University, Bahrain
}

\begin{abstract}
The accuracy and reliability of the wrist oscillometry meter was determined by both qualitative and statistical procedure. The mercury sphygmomanometer was taken as the standard of all blood pressure measurements in this study. Blood pressures from the author were both measured by the mercury sphygmomanometer and wrist oscillometry meter. The data show that the wrist oscillometry meter is giving a variability in the measurements that makes it difficult to be used as a blood pressure meter compared with the mercury sphygmomanometer. More specifically, it is not possible to ascertain in one measurement whether the patient's blood pressure is low or high. This was shown qualitatively and quantitatively using statistical procedure (t- test and Monte Carlo simulation).
\end{abstract}

Keywords: Blood Pressure, Wrist, Oscillometry, Oscillometric method, Error, Measurement

\section{INTRODUCTION}

People nowadays are becoming more health conscious and blood pressure measurement monitors are becoming more and more seen in the household and the toxicity issue of the mercury sphygmomanometer makes it necessary to make available another method of blood pressure measurement.

There are now many available apparatus in the market that advertise good accuracy and easy to use instruments. But are they really good enough for the people to rely on? Published studies available show conflicting interpretations and conclusions. Therefore the methodology and data as presented in this research should be analyzed for possible use by health care givers and the public in general.

\subsection{Statement of the Problem}

The mercury sphygmomanometer was the most popular and considered accurate way to measure blood pressure. It has been referred to as the gold standard of blood pressure measurement. But the use of this instrument waned in popularity and in some situations banned because of the toxicity of mercury in the instrument. Because of this, there was a recent proliferation of alternative methods that do not use mercury in the instrument. However, the accuracy of these instruments have been put into questions and there are many conflicting reports that one can obtain from the published materials. Since the health of the people is involved and life and death situation is the question here, it is of prime importance to educate the public on these products as regards their accuracy and suitability for use. In the analysis of the gathered data, we need to consider also that people who frequent the clinic for blood pressure measurements are familiar with their own blood pressure so that they can also see if an instrument is not giving correct results.

\subsection{Objectives:}

(1) To describe the principle involve in the instruments that are being marketed nowadays to measure blood pressure.

(2) To determine the accuracy and reliability of the wrist oscillometry blood pressure meter.

(3) To recommend a procedure in the determination of the suitability of a particular blood pressure monitor.

\subsection{Scope and Limitation}

Since the two most commonly used methods of blood pressure measurement are the mercury sphygmomanometer using the korotkoff sound from the arm pulse, and the oscillometric wrist pressure measurement, the focus of this study will be on those two methods. The variability with respect to factors such as age, weight, or nervousness of the patient were not included but it was the accuracy difference between the two methods in the measurement of blood pressure that was given emphasis. The study focused on the suitability of wrist oscillometric method to give a precise reading in at least one trial of measurement just like in the case of the mercury or analog blood pressure manometers. Familiarity in the output of the mercury sphygmomanometer was assumed in the analysis of the data. People who have measuring blood pressure for a long time could easily detect accuracy differences of two instruments by comparison even without statistics. 


\section{International Advanced Research Journal in Science, Engineering and Technology}

Vol. 6, Issue 1, January 2019

\section{LITERATURE REVIEW}

\subsection{What is High Blood Pressure?}

High blood pressure is also called hypertension. It is described as a blood pressure higher than 140/ 90 where 140 is the systolic blood pressure the pressure of the blood when the blood is pumping and 90 is the diastolic pressure which is the pressure of the blood when the heart is at rest [1].

Ten percent of adults are afflicted with high blood pressure, and 50\% of all people of age 65 or more have high blood pressure. High blood pressure has many side effects such as cardiovascular disease and kidney failure as the two most severe effect of high blood pressure. It can also cause headaches and instability. The fundamental cause of high blood pressure is the abnormal force exerted by the heart in pumping blood and the resistance offered by the arteries to the blood flow. There are many causes of high blood pressure: high salt (sodium) content in the diet, weight, hereditary, alcohol consumption, smoking and age. It is actually the average pressure that defines high blood pressure because blood pressure can fluctuate from hour to hour [2].

Cerebrovascular mortality is the most common cause of death from high blood pressure. Anyone can have high blood pressure regardless of race [3].

\subsection{Types of High Blood Pressure}

High blood pressure can be classified as essential or primary or secondary, reno vascular, labile, malignant and resistant [4].

If the cause of the high pressure cannot be found it is called primary and if the cause can be found it is called secondary. If the cause can be found then the high blood pressure can be controlled [4].

Renovascular high blood pressure is due to the narrowing of the arteries supplying the kidneys. This narrowing is cause by the accumulation of cholesterol in the blood. The kidney responds by releasing hormones that increases the salt content of the blood, this is the one that causes high blood pressure [4].

Labile high blood pressure is the fluctuation in the level of blood pressure. This is due to the emotional stress.

Malignant hypertension is a very high blood pressure (180/120). It develops very rapidly and may cause damage to organs of the body.

Resistant hypertension is a kind of blood pressure that remains high even after the application of three different types of antihypertension. Systolic blood pressure remains high.

\subsection{Methods of Blood Pressure Measurements}

\section{Auscultatory Method}

The mercury sphygmomanometer was the most popular and accurate instrument used in the measurement of blood pressure but its popularity has largely diminished because of is dependence on the use of mercury for pressure measurement. Mercury is a toxic liquid metal and its use is banned in many countries. This instrument measures the pressure in the brachial artery by listening to the Korotkoff sound using a stethoscope. It could be difficult if the patient has faint Koroskoff sounds. The mercury sphygmomanometer is replaced now by aneroid manometers; they are less accurate and need frequent calibration [5].

\section{Oscillometric Method}

This is based on the measurement of blood pressure oscillations from the appearance of the first Korotkoff sound to the last Korotkoff sound. The blood pressure during the maximum oscillation corresponds to the average blood pressure. The systolic and the diastolic blood pressures are then estimated by a suitable algorithm. This algorithm varies in different equipment. This is method is considered very accurate. It does not require a stethoscope to listen to the Korotkoff sound and thus the blood pressure can be measured even if it is noisy [6].

\section{Ultrasound Method}

The onset of blood flow when the sphygmomanometer cuff is released is detected by the shift in the frequency of the reflected sound (Doppler Effect) when the arterial wall is targeted by an ultrasound transmitter. The pressure at this point is equivalent to the diastolic pressure as measured by the Korotkoff sound. This is an alternative solution to patients with very faint Korotkoff sounds.

\subsection{Accuracy of Blood Pressure Measurements}

Only the ausculatory method and the oscillometric method will be discussed since the focus of this research is in the comparison between the method using the Korotkoff sounds and the method using the oscillation of the blood pressure particularly the use of the wrist monitors. They are also the most common methods of blood pressure measurement. 


\section{International Advanced Research Journal in Science, Engineering and Technology}

Vol. 6, Issue 1, January 2019

There has been reports that the wrist monitors are not accurate [7], however some researches that have been published concluded that they are reasonably accurate [8].

In the work of Menezes [8], parallel blood pressure measurements were made between a wrist digital sphygmomanometer and a mercury sphygmomanometer. A total difference of 20 units were obtained for both systolic and diastolic blood pressures. In terms of standard deviation this is about 10 units. A difference of this magnitude can make blood pressure measurements impossible to be determined in one or two measurements. In the use of the mercury sphygmomanometer, it is usually possible even with one trial of measurements to obtain with confidence the value of both the systolic and diastolic measurements.

\subsection{Errors in Measurement of Blood Pressure}

\subsubsection{The White Coat Effect}

There are instances wherein the patient's blood pressure is elevated in the presence of the doctor or the nurse taking the blood pressure. This is due to the anxiety of the patient [9]. Some patients may even show an elevated blood pressure no matter how long they stay in the clinic. This effect is very observable to hypertensive patients and seldom observed in patients with normal blood pressure.

\subsubsection{Speed of Cuff Inflation or Deflation}

The speed of cuff inflation has no significant effect on the detection of korotkoff sound but the speed of deflation if too slow can result to a high reading of the diastolic pressure. The ideal speed of deflation is 2 to $3 \mathrm{mmHg}$ per second.

\subsubsection{Calibration of the manometer}

A mercury column must read zero when it is completely deplated. The mercury column should also be cleaned so that it flows freely during deflation, otherwise erroneous reading will be obtained. Aneroid meter should be checked regularly against a mercury sphygmomanometer since they could be inaccurate [10].

\subsubsection{Error in Oscillometric Blood Pressure Measurement}

Error can result if the meter is not at the same level as the heart. This is prevalent to oscillometric methods of blood pressure measurement. This is attributed to hydrostatic effect [11].

\subsubsection{Practice in the Measurement of Blood Pressure}

Errors in the measurement may also be due to the bad practice of clinic personnel. It has been found in a survey that less than $1 \%$ of clinic personnel are following the American Association Guidelines in the measurement of blood pressure and that $60 \%$ are actually measuring blood pressures inaccurately [12].

\section{METHODOLOGY}

A procedure was created in determining the reliability of a wrist oscillometry blood measure monitor. The usefulness of this monitor based on common usage of a typical blood pressure monitor was evaluated. That is, in one measurement we should be able to determine whether the measured blood pressure is substantially high (or low) to merit taking medication. The procedure used also utilized the principles of Monte Carlo simulation.

\subsection{Collection of Blood Pressure Measurements}

Blood pressure measurements were taken from the author. This was contrary to other published researches where data were taken from different people to be able to create many different variables such as age, race, nationality, sex, etc. In this research, since the data was from one person only, all those were not considered so that only the most important variables were considered such as accuracy and type of monitor. This does not limit the usefulness of the procedure since anyone can always do his own evaluation of the instrument for his own personal use.

\subsection{Monte Carlo Simulation}

The procedure for the Monte Carlo simulation boiled down here to a simple concept. It involved identifying the variables in the input and the prediction of the output from a defined function. Samples were taken and a normal distribution was used to describe the input variable. And then more input values of the variable were generated using the same normal distribution. Then from those generated inputs analyses were then applied.

The Monte Carlo simulation gives us a range of unlimited possible values for our variables based on minimal data that we have. By using Monte Carlo simulation we can generate thousands of data where a more direct and simple analysis 


\section{International Advanced Research Journal in Science, Engineering and Technology}

Vol. 6, Issue 1, January 2019

can be used. This also minimizes efforts and expenses in the gathering of data samples. Many statistical distributions are used in Monte Carlo Simulations but the normal distribution is the most commonly used.

\subsection{Methods of Analysis of the Data}

The procedure can be taken from the works of Mohan [13].

In the collection of blood pressure data two levels of a single variable were used: low and high levels. Samples were taken at low level and another set of samples at high level. From any of these two sets, the mean and standard deviation were computed. Normal distribution was used to describe the values. With the two levels now defined, more inputs were predicted. An example of the simulation. An input was generated using the normal distribution for a given average blood pressure level say 130 if the input say generated is 140 then it definitely is not reliable because 130 is acceptable but 140 is too high, even a reading of 135 is already considered too high. So that we can see that if the generated inputs are five degree higher or lower then it is not acceptable. In other words if the actual level of blood pressure is 130 and by simulation we are getting 5 degrees higher or lower than the instrument is not accurate enough for use since it is indicating low or high readings that the actual true value.

Another way to look at it. Based on the simulation, we are getting say 9 values within the 125 to 135 range and just one value outside this range, then the instrument accuracy is acceptable, it is $90 \%$ within the correct range range and there is $10 \%$ probability or erroneous reading. It should actually be better than this. Please take note that those who have been measuring blood pressure for a long time can have a feel of whether the instrument is reasonably accurate or not even without this statistical analysis. So the result of this research was more on quantification.

\subsection{Absolute Readings of the Monitor Under Study}

Usually the monitor in study would give a mean reading above or below that of the one obtained by using the mercury sphygmomanometer. There was no problem with this. All we did was to translate the values. For example if the actual mean was $130 \mathrm{mmHg}$ (using the mercury sphygmomanometer) for the low level of blood pressure and the mean of the monitor under study was $150 \mathrm{mmHg}$ then we simply considered the 150 mean reading as our low level of blood pressure using the monitor under study. The same thing applied to the high blood pressure level.

\section{RESULTS AND DISCUSIONS}

\subsection{Systolic Blood Pressures as Measured by Wrist Monitor}

Table 4.1 Systolic blood pressure (SBP) high and low levels as measured by wrist oscillometric monitor with the measured value (standard) from the mercury sphygmomanometer.

\begin{tabular}{|l|l|}
\hline $\begin{array}{l}\text { SBP set as 150 mmHg by } \\
\text { mercury sphygmomanometer }\end{array}$ & $\begin{array}{l}\text { SBP set as } 140 \text { mmHg by } \\
\text { mercury sphygmomanometer }\end{array}$ \\
\hline 169 & 178 \\
\hline 175 & 169 \\
\hline 172 & 171 \\
\hline 168 & 173 \\
\hline 168 & 168 \\
\hline 177 & 155 \\
\hline 175 & 171 \\
\hline 177 & 157 \\
\hline
\end{tabular}

Table 4.2 Diastolic blood pressure (DBP) high and low levels as measured by wrist monitor

\begin{tabular}{|l|l|}
\hline $\begin{array}{l}\text { DBP set as } 90 \mathrm{mmHg} \text { by mercury } \\
\text { sphygmomanometer }\end{array}$ & $\begin{array}{l}\text { DBP set as 85 mmHg by mercury } \\
\text { sphygmomanometer }\end{array}$ \\
\hline 101 & 106 \\
\hline 106 & 106 \\
\hline 100 & 108 \\
\hline 100 & 100 \\
\hline 106 & 97 \\
\hline 102 & 96 \\
\hline 101 & 93 \\
\hline 106 & 95 \\
\hline
\end{tabular}




\section{International Advanced Research Journal in Science, Engineering and Technology}

Vol. 6, Issue 1, January 2019

In Table 4.1, we can use t-test to determine to test for the significance in the measured mean of SBP between and high and low levels. The result is shown below.

t-Test: Paired Two Sample for Means

\begin{tabular}{lll}
\hline & Variable 1 & Variable 2 \\
\hline Mean & 172.625 & 167.75 \\
Variance & 15.125 & 61.92857 \\
Observations & 8 & 8 \\
Pearson Correlation & -0.73167 & \\
Hypothesized Mean Difference & 0 & \\
df & 7 & \\
t Stat & 1.249183 & \\
P(T<=t) one-tail & 0.125875 & \\
t Critical one-tail & 1.894579 & \\
P(T<=t) two-tail & 0.251749 & \\
$\mathrm{t}$ Critical two-tail & 2.364624 & \\
\hline
\end{tabular}

We can see that using the one tailed test with a p-value of $0.126(0.05)$, there is no significance between the means. It shows that for that six pairs of samples the wrist monitor was not able to detect a difference. Whereas there is actually a difference as measured by the mercury sphygmomanometer. In other words the difference of 150-140=10 was not detected by the wrist monitor.

We can do the same thing for the DBPs. The result of the t-test is shown below.

t-Test: Paired Two Sample for Means

\begin{tabular}{lll}
\hline & Variable 1 & Variable 2 \\
\hline Mean & 102.75 & 100.125 \\
Variance & 7.642857 & 33.55357 \\
Observations & 8 & 8 \\
Pearson Correlation & -0.21187 & \\
Hypothesized Mean Difference & 0 & \\
df & 7 & \\
t Stat & 1.071851 & \\
P(T<=t) one-tail & 0.159672 & \\
t Critical one-tail & 1.894579 & \\
P(T<=t) two-tail & 0.319344 & \\
t Critical two-tail & 2.364624 & \\
\hline
\end{tabular}

Again the p-value obtained using the one-tailed test was $0.160(<0.05)$. It means that the wrist monitor failed to determine a difference between the means. The actual difference of $90-85=5$ was not detected by wrist monitor for the 6 pairs of values used.

\subsection{Monte Carlo Simulation}

Here, the two columns of Table 4.1 were combined since there was no significant difference between them. The mean and standard deviation of the SBP values were computed as 170.3 and 6.15 respectively. From these mean and standard deviation, point values of the SBP can be computed using Microsoft Excel's formula NORMINV(RAND). If there is a 5 unit difference between the point value and the mean it is indicated as OK or acceptable, $\mathrm{L}$ or low and $\mathrm{H}$ or high in Table 4.3. The wrist monitor to be acceptable all the readings should be OK so that in one measurement it is possible to be certain whether the blood pressure as measured is high or low. 


\section{International Advanced Research Journal in Science, Engineering and Technology}

Vol. 6, Issue 1, January 2019

Table 4.3 Monte Carlo Simulation of the SBP values

\begin{tabular}{|l|l|}
\hline Point value & Evaluation \\
\hline 171.131 & OK \\
\hline 157.119 & L \\
\hline 165.7866 & OK \\
\hline 174.2067 & OK \\
\hline 173.7034 & OK \\
\hline 164.8094 & L \\
\hline 172.985 & OK \\
\hline 180.1529 & H \\
\hline 160.8633 & L \\
\hline 173.5616 & OK \\
\hline 164.0708 & L \\
\hline 158.4125 & L \\
\hline 167.6611 & OK \\
\hline 174.3123 & OK \\
\hline 170.6495 & OK \\
\hline 171.2864 & OK \\
\hline 172.9511 & OK \\
\hline 168.5224 & OK \\
\hline
\end{tabular}

\section{CONCLUSION}

The wrist oscillometry meter exhibits a rather large variability in the measurement of blood pressure for it to be of any practical use. The data also indicates that it fails to differentiate between low and high levels of blood pressure (difference of $10 \mathrm{mmHg}$ ), both in the systolic and diastolic blood pressures (t-test, $5 \%$ level of significance). Variability (absolute deviation) of higher than $\pm 5 \mathrm{mmHg}$ as shown in the Monte Carlo Simulation will not make it possible to measure the blood pressure in one trial which is possible to do using the mercury sphygmomanometer. The methodology described in this research may be used in the evaluation of new monitors that are being introduced into the market. The evaluation methods available in the literature is definitely not recommended because the data may show correlations between true and measured values but the monitor may fail to give the required sensitivity or resolution to be of practical use. The medical professional groups and societies should look into this matter more seriously because this may jeopardize the health of people that rely on this kind of equipment for monitoring their blood pressure. Nowadays a lot of products which are useless and not working are being marketed endlessly. It is amazing how such a thing can be perpetuated without triggering a scientific investigation.

\section{REFERENCES}

[1]. WEBMD. (05 March 2017). Retrieved from http://www.webmd.com/hypertension-high-blood-pressure/guide/diastolic-and-systolic-bloodpressure-know-your-numbers

[2]. Fahey T, Murphy D. (2004). High Blood Pressure, Class Publishing (London) Ltd.

[3]. Kesteloot H, Joosens J V. (2012). Epidemiology of Arterial Blood Presssure, Springer Science \& Business Media

[4]. Chhajer B. (2014). High Blood Pressure, Control and Cure, Diamond Pocket Books Pvt Ltd, Aug 13, 2014 - 32 pages

[5]. Pickering TG, Hall JE, Appel LJ, et al. (2005). Recommendations for blood pressure measurement in humans and experimental animals: Part 1: blood pressure measurement in humans: a statement for professionals from the Subcommittee of Professional and Public Education of the American Heart Association Council on High Blood Pressure. Research Hypertension; 45:142.

[6]. Ogedegbe G, pickering T. (2010). Principles and techniques of blood pressure measurement. Cardiol Clin., November; 28(4): 571-586.

[7]. Doshi H, Weder A, Bard R, Brook R. (2010). Does "hidden undercuffing" occur among obese patients? Effect of arm sizes and other predictors of the difference between wrist and upper arm blood pressures. Journal of Clinical Hypertension.12:82-88.

[8]. Menezes A, Dumith S, Noal R, Nunes A, Mendonza I, Araujo C, Duval M, Caruso P, Hallal P. (2009). Validity of wrist digital monitor for blood pressure measurement in comparison to a mercury sphygmomanometer. Arc Bras Cardiol 2010; 94(3): 345-349.

[9]. Jhalani J, Goyal T, Clemow, et al. (2005). Anxiety and outcome expectations predict the white-coat effect . Blood Press Monit.; $10: 317$.

[10]. Burk MJ, Towers HM, O’Malley K, et al. (1982). Sphygmomanometer in hospital and family practice: problems and recommendations. Br Med $J$ (Clin Res Ed); 285:469.

[11]. Mitchell PL, Parlin RW, Blackburn H. (1964). Effect of vertical displacement of the arm on indirect blood-pressure measurement. $N$ Engl $J$ Med; 271:72

[12]. Villegas I, Arias IC, Botero A, et al.(1995) Evaluation of the technique used by health care workers for taking blood pressure. Hypertension

[13]. Mooney C. (1997). Monte Carlo Simulation, Quantitaive Applications in the Social Sciences. SAGE Publications, Inc.

[14]. Mohan A, Chitale R. (2016). Managing the influence of inventory bullwhip effect on profit maximization in rural retailing using Monte Carlo Simulation. The Journal - Contemporary Management Research 2016, Vol. 10, Issue No. 2, 24 - 33. 